\title{
First Reported Case of Extramedullary Plasmacytoma of the Appendix
}

\author{
Mark G. Evans ${ }^{\mathrm{a}, \mathrm{b}}$, Xiaohui Zhao ${ }^{\mathrm{a}}$, Fritz Lin ${ }^{\mathrm{a}}$, Beverly Y. Wang ${ }^{\mathrm{a}}$
}

\begin{abstract}
An extramedullary plasmacytoma involving the gastrointestinal tract is extremely rare. We report an appendiceal extramedullary plasmacytoma in a 35-year-old man who presented to the emergency department because of upper abdominal pain. Computed tomography (CT) imaging revealed an incidental mass $(3.7 \times 1.9 \times 1.6 \mathrm{~cm})$ at the tip of the appendix. Microscopically, the appendix, periappendiceal soft tissue, and nearby lymph nodes were diffusely infiltrated by plasma cells that were kappa light chain restricted. Subsequent workup included an unremarkable bone marrow biopsy, as well as urine and serum electrophoresis. A diagnosis of kappa-restricted solitary extramedullary plasmacytoma was made. To our knowledge, this is the first case reported of an appendiceal extramedullary plasmacytoma in the medical literature.
\end{abstract}

Keywords: Extramedullary plasmacytoma; Gastrointestinal tract; Appendix; Plasma cell neoplasm

\section{Introduction}

Extramedullary plasmacytoma (EMP) refers to a monoclonal proliferation of plasma cells occurring at a non-osseous site in the absence of bone marrow involvement. It accounts for only $3-5 \%$ of plasma cell neoplasms [1]. Generally found in the head and neck region, rare EMPs of the gastrointestinal tract have been reported. To date, they have been observed in the esophagus, stomach, ileum, cecum, transverse, ascending, and rectosigmoid colon $[2,3]$. To our knowledge, this study presents the first case of an EMP localized to the appendix.

\section{Case Report}

A 35-year-old man presented to a local emergency department

Manuscript submitted March 12, 2020, accepted April 10, 2020

aDepartment of Pathology and Laboratory Medicine, University of California Irvine (UCI) Medical Center, Orange, CA 92868, USA

${ }^{\text {b} C o r r e s p o n d i n g ~ A u t h o r: ~ M a r k ~ G . ~ E v a n s, ~ D e p a r t m e n t ~ o f ~ P a t h o l o g y ~ a n d ~ L a b o-~}$ ratory Medicine, University of California Irvine, 101 The City Drive South, Orange, CA 92868, USA. Email: markevans2011@gmail.com

doi: https://doi.org/10.14740/gr1277 following three episodes of nighttime upper abdominal pain. His past medical and surgical history was notable for a left inguinal hernia repair during childhood and a vague history of gastritis for several years. Computed tomography (CT) imaging revealed a single gallbladder calculus without radiographic evidence of cholecystitis and an incidental mass $(3.7 \times 1.9 \times$ $1.6 \mathrm{~cm}$ ) at the tip of the appendix (Fig. 1).

Appendectomy was performed and a tan-yellow, semisolid, homogenous nodule was detected at the tip of the appendix. On microscopy, a proliferation of plasma cells was present within the lumen and infiltrated through the muscle wall into the surrounding soft tissue (Fig. 2). Diminutive periappendiceal lymph nodes also contained the neoplastic cells. The plasma cells were kappa light chain restricted and positive for cluster of differentiation 138 (CD138) and MUM-1 by immunohistochemistry. Ki-67 demonstrated a low proliferative index of approximately $10 \%$. The surgical resection margin was uninvolved by neoplastic cells.

The overall findings were consistent with a kappa-restricted solitary EMP. An unremarkable bone marrow biopsy was performed, and laboratory testing failed to reveal monoclonal gammopathy by serum and urine electrophoresis. The patient was recommended no further treatment, and he is currently alive without evidence of disease.

\section{Discussion}

Gastrointestinal EMPs are rare and account for up to only $10 \%$

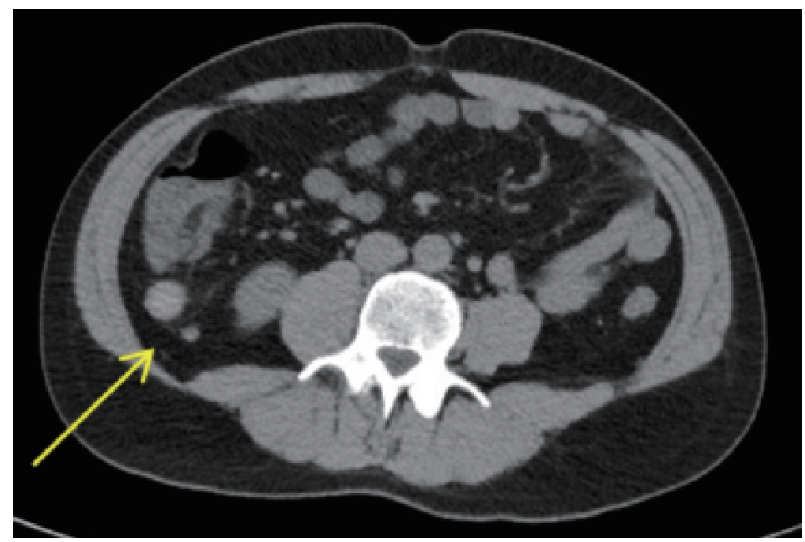

Figure 1. Abdominal CT imaging demonstrated a $3.7-\mathrm{cm}$ mass at the appendiceal tip (arrow). CT: computed tomography. 


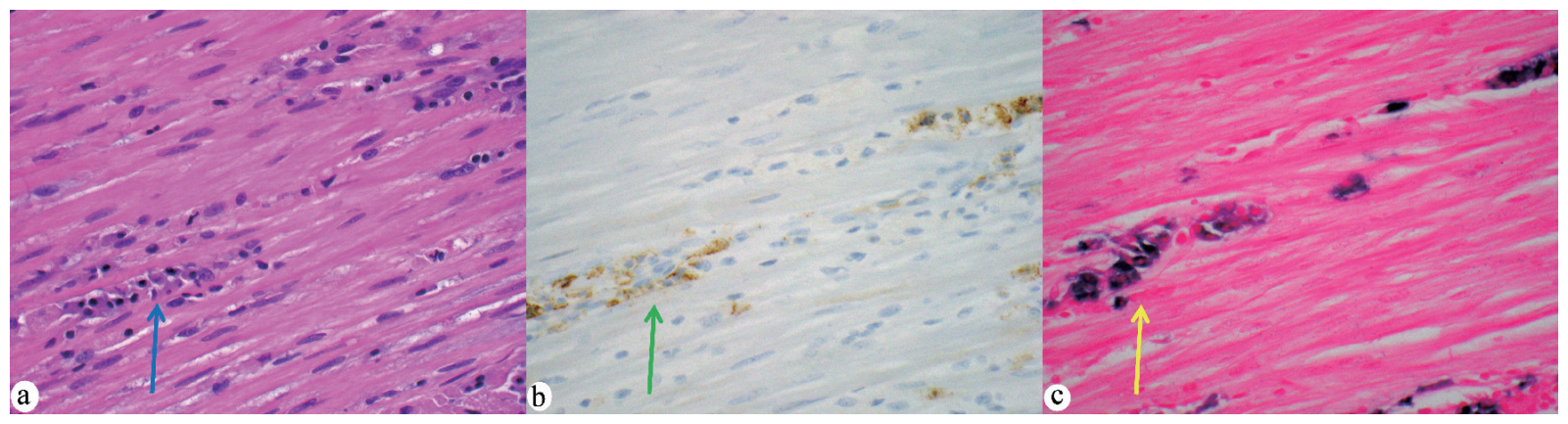

Figure 2. (a) The appendiceal wall was infiltrated by a plasma cell neoplasm (hematoxylin and eosin staining, $\times 400$, plasma cells indicated by blue arrow). (b) The cells were positive for cluster of differentiation 138 (CD138) (anti-CD138 immunohistochemistry, $\times 400$, positive cells indicated by green arrow). (c) The cells also demonstrated prominent kappa light chain restriction (kappa in situ hybridization, $\times 400$, positive cells indicated by yellow arrow).

of cases [4]. They can be classified as primary and secondary, depending on whether the patient has a history of previous plasma cell neoplasm. Secondary cases are more uncommon, and the prevalence of EMP in patients with newly diagnosed multiple myeloma (MM) ranges from $2 \%$ to $20 \%$ [5]. Fewer than 25 cases of secondary plasmacytomas have been reported in the gastrointestinal tract. In cases of primary EMP, approximately one-third progress to MM within 2 years of symptom onset [6]. Follow-up with repeat biopsies, imaging, and electrophoresis is therefore highly recommended.

EMP typically has a better prognosis than other plasma cell neoplasms. Five-year survival is estimated at $82 \%$, with 10 -year disease-specific survival greater than $50 \%[7,8]$. Several treatments have been proposed, including radiation, chemotherapy, and surgical removal. EMPs are radiosensitive, and radiation therapy is advocated in the head and neck region. The United Kingdom Myeloma Forum however recommends initial surgery for cases at other anatomic sites [9]. Endoscopic mucosal resection (EMR) or endoscopic submucosal dissection (ESD) is generally performed for gastrointestinal EMPs. Chemotherapy is generally reserved for large, higher-grade tumors or following recurrence or local treatment failures.

Some studies suggest that Helicobacter pylori (H. pylori) infection may ben associated with EMP, particularly at gastric sites. Zhang et al reported an ileocecal plasmacytoma in the setting of $H$. pylori-gastritis [9]. Our patient had a vague history of non-specific gastritis for several years, which could be suspicious for bacterial infection. However, gastric biopsies and serologic testing performed following appendectomy were negative for $H$. pylori and revealed only mild chronic gastritis.

EMPs of the gastrointestinal tract typically present with non-specific abdominal pain. Rectal bleeding, nausea, vomiting, anorexia, bowel obstruction, changes in bowel habits, and intussusception have also been reported [10]. These symptoms should prompt radiographic imaging, which will demonstrate well-defied soft tissue masses on CT scan and heterogenous enhancement on magnetic resonance imaging (MRI). While rare, EMP should still be considered within the differential diagnosis for such lesions. Treating physicians should then proceed to biopsy or complete surgical excision, as was performed for our patient, who currently demonstrates no evidence of disease.

\section{Acknowledgments}

We would like to acknowledge the support of the Anatomic Pathology Section of the Department of Pathology and Laboratory Medicine at the University of California, Irvine.

\section{Financial Disclosure}

No sources of funding or financial contributions to declare.

\section{Conflict of Interest}

None to declare.

\section{Informed Consent}

Informed consent was obtained by the treating physicians.

\section{Author Contributions}

Mark G. Evans contributed to the conception and drafting of the article. Xiaohui Zhao contributed to the revising of the article. Fritz Lin contributed to the revising of the article. Beverly Y. Wang contributed to the conception and revising of the article.

\section{Data Availability}

The authors declare that data supporting the findings of this study are available within the article.

\section{References}

1. Nolan KD, Mone MC, Nelson EW. Plasma cell neoplasms. Review of disease progression and report of a new variant. Surg Oncol. 2005;14(2):85-90. 
2. Mjoli M, Vorajee N, Naidoo Y, Madiba T. Solitary extramedullary plasmacytoma of the colon, rectum and anus. S Afr J Surg. 2016;54(2):45-47.

3. Han YJ, Park SJ, Park MI, Moon W, Kim SE, Ku KH, Ock SY. Solitary extramedullary plasmacytoma in the gastrointestinal tract: report of two cases and review of literature. Korean J Gastroenterol. 2014;63(5):316320 .

4. Dalgic T, Bostanci EB, Cakir T, Ozer Il, Ulas M, Aydog G, Akoglu M. Solitary plasmacytoma of the cecum and the ascending colon: surgical resection as a treatment modality. Case Reports in Surg. 2015;2015:1-4.

5. Parnell K, Ahmed M, Smalligan RD, Nadesan S. Extramedullary plasmacytoma mimicking colon carcinoma: an unusual presentation and review of the literature. BMJ Case Rep. 2015;2015.

6. Ahnach M, Marouan S, Rachid M, Madani A, Quessar A,
Benchekroun S, Quachouh M. Extramedullary plasmocytoma relapsing at differents sites: an unusual presentation. Pan Afr Med J. 2013;14:34.

7. Webb CJ, Makura ZG, Jackson SR, Helliwell T. Primary extramedullary plasmacytoma of the tongue base. Case report and review of the literature. ORL J Otorhinolaryngol Relat Spec. 2002;64(4):278-280.

8. Straetmans J, Stokroos R. Extramedullary plasmacytomas in the head and neck region. Eur Arch Otorhinolaryngol. 2008;265(11):1417-1423.

9. Zhang D, Cao D, Shen D, Mulmi Shrestha S, Yin Y. Extramedullary plasmacytoma occuring in ileocecum: A case report and literature review. Medicine (Baltimore). 2017;96(51):e9313.

10. Lee SH, Ahn BK, Baek SU, Chang HK. Primary isolated extramedullary plasmacytoma in the colon. Gastroenterology Res. 2013;6(4):152-155. 